(C) 2011 IEEE. Personal use of this material is permitted. Permission from IEEE must be obtained for all other uses, in any current or future media, including reprinting/republishing this material for advertising or promotional purposes, creating new collective works, for resale or redistribution to servers or lists, or reuse of any copyrighted component of this work in other works. 


\title{
Identifying Prosumer's Energy Sharing Behaviours for Forming Optimal Prosumer-Communities
}

\author{
A.J. Dinusha Rathnayaka, Vidyasagar M. Potdar, Omar Hussain, Tharam Dillon \\ Curtin University of Technology \\ Perth, Australia \\ Abekoon.rathnayaka@postgrad.curtin.edu.au
}

\begin{abstract}
Smart Grid (SG) achieves bidirectional energy and information flow between the energy user and the utility grid, allowing energy users not only to consume energy, but also to generate the energy and share with the utility grid or with other energy consumers. This type of energy user who consumes energy and who also can generate the energy is called the "prosumer". The sustainability of the SG energy sharing process heavily depends on its participating prosumers, making prosumer participation and management schemes crucial within the energy sharing approaches. However in literature, there is very little attention on prosumer management schemes. The contribution of this article is twofold. First, this article introduces a novel concept of participating and managing the prosumers in the SG energy sharing process in the form of autonomous, intelligent goal-oriented virtual communities. Here, the community of prosumers can collectively increase the amount of power to be auctioned or bought offering higher bargaining power, thereby settling for a higher price per kilowatt in long-term. According to the literature, this research is the first of this type introducing a community approach for prosumer management. The initial step to build an effective prosumer-community is the identification of those prosumers who would be suitable to make efficient prosumer communities. This leads the necessity of identifying parameters that influence the energy sharing behaviours of prosumers. The second contribution of this article is that, this comprehensively analyzes the different parameters influencing the prosumer's energy sharing behaviours and thus presents multi-agent architecture for optimal prosumer-community formation.
\end{abstract}

Keywords- smart grid; energy sharing; energy behaviour; prosumer; prosumer-profiling; multi agent system

\section{INTRODUCTION}

The demand for energy in the world is continually rising. Worldwide energy consumption is expected to increase by 49 percent from 2007 to 2035[1]. The majority of the current demand is met by non-renewable energy sources like coal, petroleum, natural gas etc., but using such non-renewable energy sources contributes to greenhouse gas emissions and global warming. In today's world, the focus is more on shifting the energy usage of users from non-renewable sources to renewable sources of energy such as solar, water, and wind. Further, users are being encouraged not only to reduce their household energy consumption, but also to generate renewable energy and either to store the excess energy for future usage or to return the excess energy back to the utility grid. In order to achieve such a framework, the vision of SG has been proposed in literature. Smart Grid (SG) is defined as "an electricity network that can intelligently integrate the behaviour and actions of all users connected to it - generators, consumers and those that do both (prosumers) - in order to deliver sustainable, economic and secure electricity supplies" [2]. SG integrates ICT (Information Communication Technologies) with advanced power electronic technologies to achieve a bidirectional flow of electricity and information [3]. It also exhibits numerous desirable characteristics such as selfhealing, high reliability, increased efficiency, improved power quality, resistance to cyber attacks, and user friendliness [4].

In order for the SG energy sharing framework to work seamlessly, it requires the effective integration of different key elements namely; (i) smart devices, (ii) bi-directional communication, (iii) software infrastructure and (iv) dynamic prosumer base. In literature, there are considerable amount of research on fulfilling the first three elements discussed above [5-8]. However, there has been very little investigation in developing schemes to build a dynamic prosumer base and those existing schemes are also not very effective in addressing some vital issues like achieving sustainability, developing effective motivation schemes, etc. In order to address this issue, in this research, we first introduce a novel approach to participate the prosumers to the $\mathrm{SG}$, which integrates the prosumers to the SG as prosumer-communities. According to the existing body of literature, there is no published work on introducing a community based framework to manage the prosumers in the SG energy sharing process. Please note that some works use the word "community" to indicate the entire prosumer base living in specific region or country; but the concept we introduce in this article is different from that. In order to form an efficient prosumer-community, the initial step would be to cluster the prosumers having mutual interests. This requires the clear identification and analysis of prosumer's diverse energy behaviours. Although there are several research efforts that investigate the behaviours of energy-user, all those methods have failed to consider some vital factors specific to energy sharing such as energy auctioning possibilities, stored quantity of energy and shared quantity of energy, possible energy buyers, etc. In this article, we analyse the factors that directly and indirectly influence the prosumers' energy sharing activities, and finally present a multi-agent architecture for prosumer-community formation. 


\section{PROSUMER PARTICIPATION IN SMART GRID}

We discuss this section under three different subsections. First, we briefly discuss the prosumer participation and management schemes used by existing energy sharing approaches and identify their shortcomings. Second, we explain the new approach, the community-based approach for the prosumer participation and management. Third, we discuss the background information on energy-user behavior analysis, since the initial groundwork to form effective prosumercommunities is analyzing different energy behaviours of prosumers.

\section{A. Literature on Prosumer Participation and Management}

Literature integrates the prosumers to the SG using two main methods; (i) individual integration, and (ii) simple-group integration in the form of a VPP (Virtual Power Plant) (Fig.1). Individual integration method links the individual prosumers to the SG, facilitating the direct energy sharing between the prosumers and the utility grid. Here the energy sharing decisions are made based on the individual perceptions [9-12].

Another method is to connect a group of prosumers to the utility grid. When it comes to energy auctioning, this collaboration becomes a Virtual Power Plant (VPP). Literature recognizes VPP as "a technology for market visibility of many small distributed energy resources" [13]. The VPPs are managed to interact, negotiate or collaborate with other participants of the utility grid to enable joint coordination about the energy usage within the group of houses and thus attain enhanced overall energy efficiency [14-17]. Integrating group of DERs (Distributed Energy Resources) attain the critical mass essential to actively participate to the energy market. As illustrated in Fig. 1, these simple prosumer groups presented in literature contain prosumers having diverse behaviours (e.g. different energy sources, different amounts of energy supplied to the grid, etc.), and those prosumers collectively increase the amount of power to be auctioned to the grid. The group of prosumers can attain the minimum amount of energy requested by the utility companies with lesser efforts when compared to the individual prosumer participation methods. However since these groups are not provoked to achieve a proper energy goal, in some cases those simple groups may fail even to achieve the minimum energy threshold accepted by the utility provision.

The existing research approaches exhibit several shortcomings, which imply the need of a new approach for prosumer participation and management. All the existing prosumer-group approaches merely aggregate the prosumers as groups, without considering their diverse behaviors. Such completely different behaviors can lead to misunderstandings and disputes among the prosumers within the same group. Since there are no clear identification and classification of prosumer behaviors, it is difficult to assess and resolve negative behaviors of prosumers such as the prosumer supplies lower amount of energy than agreed in the VPP formation process. These behaviors can affect adversely to all the members of the prosumer-groups. In addition these existing groups are not provoked to achieve proper goals, which can result ad-hoc pattern of energy supply to the grid, thus there may not be a guaranteed sufficient energy supply all the time. Another shortcoming is that currently there is no comprehensi-

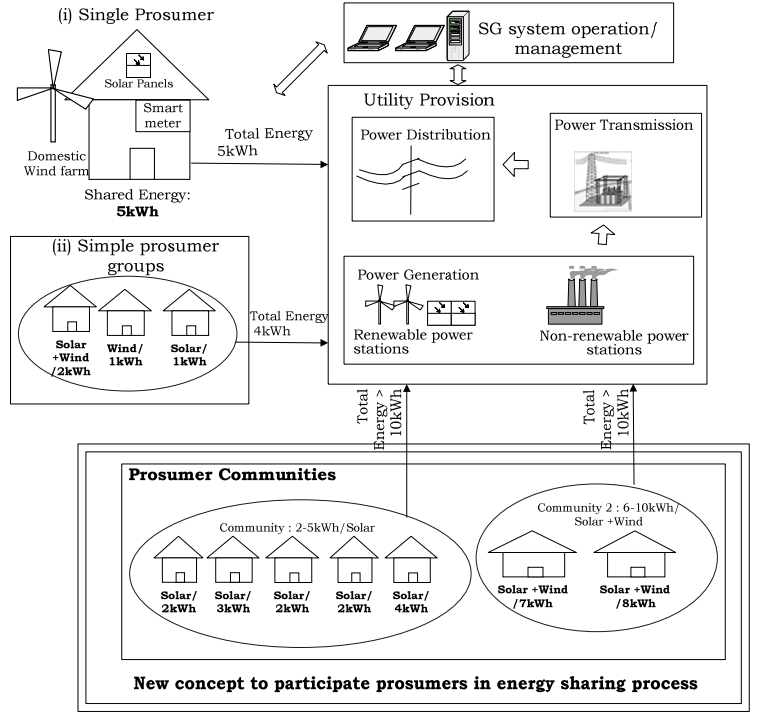

Fig. 1. Prosumer participation and management schemes

-ve approach to identify and attract more dynamic prosumers and grow the prosumer base. This is important because relying on the minimum number of prosumers is short-sighted in SG energy sharing, due to the uncertainty involved with climate dependant green energy sources and prosumer behaviors.

In order to address these issues, we introduce a new concept of integrating prosumers in the form of prosumer communities, rather than individual prosumer integration and simple prosumer group integration (Fig. 1).

\section{B. The Concept of Prosumer-communities}

In this section, we explain the new concept of 'prosumercommunity', as an approach to participate and manage the prosumers in the SG energy sharing process. This method connects the prosumers to the SG in the form of goal-oriented virtual communities. The community of prosumers interacts with each other and with the local distributor or the utility grid through the community gateway, which is a smart intermediate and intelligent component that bridges the utility grid with smart devices of each distributed energy resource that belong to the community. Further implementation details are out of the scope of this paper.

In fact, prosumer community is a major improvement to the simple prosumer groups. Unlike the simple prosumer groups, the prosumer-communities are established aggregating prosumers having similar interests and behaviours (e.g. similar energy sources, similar amount of energy supplied the grid, etc.) and those communities are stimulated to achieve a common goal (e.g. to achieve at least the minimum amount of energy requested by utility provision, the minimum amount of energy required to obtain higher bargaining power in the energy market, etc.). One way to obtain prosumer-communities can be illustrated using Fig.1. In Fig. 1, the prosumers who offer energy between $2-5 \mathrm{kWh}$ through their domestic solar 
panels will be added to one community and the prosumers who offer energy between $6-10 \mathrm{kWh}$ will be added to another community. The communities are provoked to achieve minimum energy of $10 \mathrm{kWh}$, which is the community goal and the number of members of the communities may be varied accordingly. Since the prosumer-community can produce higher quantity of collective energy in long-term compared to the existing approaches, the communities can offer higher bargaining power for its members in long-term. In addition, since the members of each community exhibit similar behaviours, it makes the incentive distribution and maintenance process easier, and reduces contradictions among members, hence leads to sustainable communities.

However in order to build an efficient prosumer community, the initial building block would be to identify and analyse the different parameters that influence the energy sharing behaviours of prosumers and choose the prosumers having similar energy behaviours to make the optimal prosumer-communities. This analysis also contributes to find out the more dynamic prosumers within the prosumer-base and also to classify the prosumers' positive and negative behaviours within the energy sharing process.

\section{Literature on Energy-user Behavior Analysis}

In this section, we discuss the existing literature on energyuser behavior analysis and highlight the associated shortcomings. In general, the research findings on user behaviour disclose considerable diversity. The literature has introduced three-domain architecture for the human behavior, which includes personal domain, behavioural domain and contextual domain [18], and later this concept has been adopted for the behaviour of electricity prosumer [19]. According this study, the "personal domain" is featured with individual's basic values, beliefs, and motives, etc., "behavioural domain" contains variables that capture the level of commitment to own green energy source, and flexibility of prosumers to shift their energy usage to off-peak hours, etc. and finally the "contextual domain" offers a range of attributes like individual's background (cultural, financial, educational, etc.), status (ownerships, etc.), economic context( income), social context ( rules and regulations), and environmental context (weather). On the other hand, an extensive set of influential parameters that affect the energy user profiles can be found in the prior research done by Miller et al. [20]. Some of the many parameters include: current and predicted information for electricity prices, weather conditions, and price of renewable sources, occupant appliance usage patterns, inhabitant occupancy status, forecasted usage budget limit, etc. Energyuser behavior analysis are further visible in the research performed by Ko et al. [21], who suggests a framework that provides power purchase recommendations to diverse users based on their personal characteristics. In addition, the iPower system project [22] achieves personalized services such as to adjust appliance settings according to the user preferences. Similarly, the European project AIM (A novel architecture for modeling, virtualising and managing the energy consumption of household appliances) [23] analyse the energy data in two different modes namely 'offline mode' and 'real-time mode'. Using the offline data, three different profiles namely inhabitant presence profile, temperature profile and light profile are created, and those would be useful in forecasting future behaviors and decision making. The real-time data would be used to handle sudden adjustments in energy management systems.

Reviewing the background information on energy user behavior analysis reveals that all the existing methods consider only the influential factors for general energy consumption and energy generation behaviours of energy-users; however, no work has considered some vital factors that influence the energy sharing behaviours of prosumers such as alternatives for energy sharing like energy storage capacities, technological advancements in emerging renewable energy systems that enable the prosumers to generate and share more green energy, energy trading possibilities, possible energy buyers, and future plans of prosumers that may increase or decrease the amount of shared energy, etc. In order to address this issue, in next sections we develop a framework that analyse the behaviours of a prosumer specifically in the context of energy sharing.

\section{IDENTIFICATION OF ENERGY SHARING BEHAVIORS}

As illustrated in Fig. 2, this framework demonstrates a set of parameters, which directly and indirectly influence the prosumer's energy sharing activities. In next subsections, we further analyze these two types of parameters.

\section{A. Internal Parameters}

Internal parameters can be captured within the prosumer's operating domain and directly influence the amount of energy shared with the SG. The main element that decides the quantity of energy that the prosumer can share is his energy consumption and generation activities.

1) Energy consumption and generation activities: Generally the prosumers generate green energy at their domestic environment, and consume the energy, store the energy for future usage and share the excess energy or the net energy saving with possible energy buyers such as the national utility grid, private energy retailers and other consumers. The different factors that affect the energy consumption and energy generation activities of prosumers are illustrated as follows;

a) Energy generation behaviours: Generally the prosumers generate the green energy in their domestic environments using energy sources such as solar and wind. The amount of energy generated through these sources varies with their capacities such as number of solar cells or wind turbines and their performance factors like losses. For example, The commonly used grid connected solar PV systems are rated with maximum of $1.5 \mathrm{~kW}$ and $3 \mathrm{~kW}$; however the average solar system will generate and export approximately $75 \%$ of its rated power to the grid, since there are losses (around $25 \%$ of rated value) involved with cleaning, inverting and transforming power from the solar cells to a usable form [24].

b) Power demand/power loads: The energy consumption of the prosumer is heavily dependant on the power loads operating in the prosumer's premises, which are operated based 
on the regular and irregular energy requirements of different energy end-users. Certain power loads such as light sources, printers, electric cookers can be classified as predictable and unpredictable during different periods of the day. For example, the power consumption of light sources is predicted to be null during the hours when the inhabitants are not present or sleeping. However these power loads are unpredictable in usual working hours of the inhabitants, due to the varying energy end-user demands and schedules. The energy-users can obtain power to activate these power loads from three different energy resources; (i) connecting loads to the main utility grid, (ii) connecting loads directly to domestic green energy resource (e.g. solar hot water systems) and (iii) connecting loads to the smart storage where the prosumer stores the energy generated from green energy source for future usage (e.g. electric vehicles). Accordingly, the amount of energy that the prosumer saves for sharing is increased or decreased based on the number of power loads operate at the premises and based on the which energy sources $\mathrm{s} /$ he used for those power loads.

c) Power storage behaviours: The prosumers can store a portion of energy in smart storages for their future usage such as for electric vehicles and generators, without sharing the entire amount of energy with the grid. The quantity of energy stored depends on the capacity and the performance of the storages. For example, the commonly used energy storages in Australia are lead-acid batteries, and lithium-ion batteries which are capable to deliver the energy of $30-40 \mathrm{Wh} / \mathrm{kg}$ and 80-200 Wh/kg respectively [25]. However those storages have shown poor performance and short cycle life under deep discharge cycling. Therefore in recent years, much attention has been drawn to develop new storage technologies like vanadium redox batteries, which can repeatedly charge and discharge over a long duration of time and also can discharge significant quantities of electricity rapidly [25]. However, if much energy is stored, the quantity of energy left for sharing is possibly be decreased.

d) Changes in regular background: The amount of power that the prosumer can save for sharing can be increased or decreased due to the certain planned or sudden changes of the regular background of the prosumer's operating area such as changes in occupancy status. For example if the occupants living in prosumer's premises are out of the house for a vacation, the total amount of power usage during that time will be definitely reduced. If the energy generation is unaffected, the total energy saving during that period will be increased. On the other hand, if the prosumer renovates his house with new electric appliances such as high wattage air conditioners, the total power usage increases and hence the net energy saving decreases.

e) Prosumer's preferences/opinions: Prosumer's consent to share the energy is controlled by his own varying preferences, interests, and opinions. For example, if the prosumer prefers to store more energy for future usage, rather than sharing with the grid, it will negatively affect the energy sharing capacity. In addition, if the prosumer has negative opinion on the incentive schemes of energy sharing, there is a

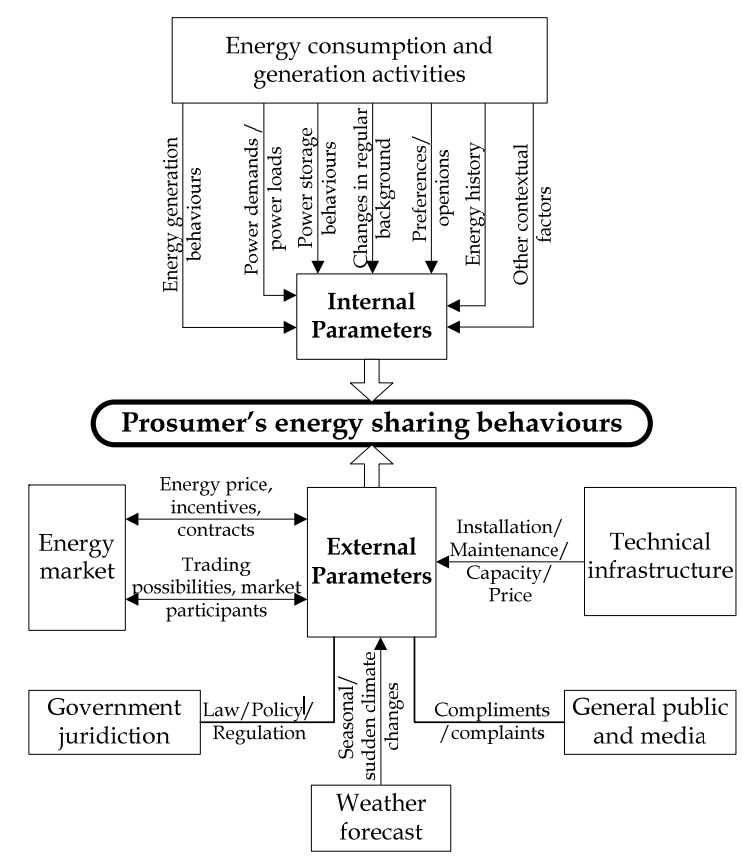

Fig.2. Identifying energy sharing behaviours of prosumers

high possibility that prosumer reduces the amount of energy shared with the grid.

f) Energy history: The prosumer's historical energy records on energy consumption and generation, and profits attained from energy sharing can offer personalized recommendations to the prosumers on how much energy should be shared in present situations and in future.

g) Other contextual factors: In addition to the above factors, there are many other contextual factors related to the prosumer that add positive or negative weights to the final amount of energy shared with the grid. Some of those factors are the prosumer's socio-cultural views, financial status and sufficient income to afford the technical infrastructures for energy sharing, availability to involve in the energy sharing process, educational knowledge on energy sharing concepts and benefits, and residential status (urban or rural) and living conditions.

\section{B. External parameters}

Unlike the internal parameters, the external parameters are captured from various different domains other than the prosumer's environment; but indirectly influence the energy sharing activities of prosumers.

1) Energy market: The energy market deals with the trade and supply of energy and commonly referred to the electricity market and also can be referred to the other energy sources as well. In this section, our focus is mainly on the electricity market. The commodities in the electricity market comprise two substances namely power and energy, where the power is 
the "metered net electrical transfer rate at any given moment" and measured in kilowatt $(\mathrm{kW})$, and the energy is "the electricity that flows through a metered point for a given period of time" and measured in kilowatt Hours (kWh) [26]. The electricity market is classified as either wholesale electricity market, where the electricity producers supply their generated electricity to retailers, or retail electricity market, where the energy users can buy the energy from preferred electricity retailer. Here we discuss how electricity market affects the energy sharing of prosumers in following factors.

2) Energy trading possibilities: The prosumer is empowered to choose the most profitable energy trading method to sell the energy in competitive energy market. For example, the prosumers can either sell the energy directly to the energy buyer or auction the energy in the auction market. Possible energy buyers would be the state government authorized national grid operators and private energy retailers in wholesale market, and private energy consumers in retail market. In direct selling, the prosumers can negotiate the price per kilowatt hour $(\mathrm{kWh})$ and the contract length with the profitable energy buyer. The other method is the auction market, which the interested buyers can send several offers to the prosumers in any given round and the wining buyer can initiate the contract with the prosumer[27]. However, so far the Western Australian (WA) prosumers cannot choose the preferred electricity buyer to sell the green energy, rather than selling to the energy retailer announced by the state government; but in the world, a numerous energy selling possibilities are open for the prosumers.

3) Electricity price, incentives, and contracts: The different energy buyers offer different energy prices, lengths of contracts and different rules and regulations in purchasing energy. The prosumers can compare the numerous options and select the most feasible scheme for them. However in WA the prosumers have no options to select, but they can take the benefits from two types of attractive incentive schemes namely; (i) feed-in tariff schemes, and (ii) Renewable Energy Buyback Scheme (REBS) [28]. The feed-in tariff scheme is funded by the state government as an incentive to stimulate the prosumers to install the renewable energy systems. At present, two feed-in tariff schemes operate in Australia namely; gross feed-in tariff and net feed-in tariff. In gross feed-in tariff scheme, a subsidy rate is assigned to all the energy that the prosumer produces, without considering the fact that whether he consumes that energy or shares that energy. In contrast, net feed-in tariff is offered only for the amount of energy shared with the grid. In WA, only the net feed-in tariff scheme operates with the rate of AUD $20 \mathrm{c} / \mathrm{kWh}$ effective from 1st July 2011. On the other hand, REBS is provided by the electricity retailers for the green energy supplied to the retailer by the prosumers. Therefore the electricity rate, contract terms and conditions may be different for different electricity retail companies. For example, in WA, the two main operators offer AUD 7 cents kWh (Synergy) and AUD 18.94 cents kWh (Horizon) under the REBS [28].
4) Government Jurisdiction: Government imposed laws, rules and regulations on energy sharing procedures can negatively or positively influence the prosumer's inspiration for energy sharing. For example, in Australia the government rules are imposed for the maximum quota for renewable systems installations, rates of government incentives, applicant's eligibility criteria, ownership of renewable systems, technical suitability of the renewable system, taxable portions of feed-in tariff income, etc. One such policy in Australia is that the individual prosumers are exempted from paying GST from their feed in tariff income, but the businesses are not. A recent example of government intervention is that by 1st of August 2011, the feed-in tariff scheme has been concluded in WA as the national grid has reached the maximum energy quota of 150MW [28].

5) Technical infrastructures: Smart grid energy sharing involves with different technical infrastructures such as renewable energy generation systems, smart metering infrastructures, smart sensors and actuators, and communication technologies. For example, in Australia, bidirectional capable meters is installed at prosumer's premises by the state government authorized agents such as Western power [29] in WA. The prosumer can choose either gross feed-in tariff meter or net feed-in tariff meter based on his operating area. The total green energy generated can be read via the inverter and also using the gross feed-in meter. The net feed-in meter only indicates the total amount of energy exported to the electricity grid since the meter was installed. In addition, different renewable energy systems are supplied by different equipment suppliers who offer different renewable energy packages suitable for diverse preferences of prosumers. These packages include different rated capacities, prices, installation and maintenance procedures, installation rebates, payment options, discounts, etc. Therefore the prosumers can choose the most feasible system for them by comparing many alternatives. Another important factor that should be considered is that these systems must meet the government technically eligibility criteria. For example, in WA, the renewable energy system should be new at the time of installation and the second hand systems will not be eligible to join the energy sharing process [28].

6) Weather forecasts: The operation of the commonly known high power consuming loads like heating and cooling loads heavily depends on the climate conditions. For example, the power consumption of the heater is higher in the cold days (winter season) and similarly air-conditioner requires more power to operate in very hot days (summer season). On the other hand, since the green energy sources are climate dependant, the amount of energy generated is increased or decreased by the weather conditions of that day. For example, the solar energy generation can be shortened in rainy days and enhanced in sunny days. For example, in Australia, the maximum quantities of electricity that the $1.5 \mathrm{~kW}$ and $3 \mathrm{~kW}$ rated solar systems generate in an hour in a average summer's day are around $1.1 \mathrm{kWh}$ and $2.1 \mathrm{kWh}$ respectively and in 
an hour of a winter day it would be around $0.9 \mathrm{kWh}$ and $1.8 \mathrm{kWh}$ respectively [24].

7) General public: The prosumers' energy sharing behaviours, especially the opinions and preferences on energy sharing are affected by the recommendations, objections, and opinions offered by other people and the media. These set of people who influence the prosumer can be his friends, relations, neighbors, teachers as well as the people unknown to the prosumer. In addition, the prosumers' behaviours can also be changed drastically due to the information transmitted by the audio and visual media such as documentaries, discussions and expert ideas on smart grid and other related issues.

As discussed in the last section, there are a number of parameters both quantitative and qualitative that directly and indirectly influence the prosumers' energy sharing behaviours. The quantitative effect of these parameters can be identified as an increase or a decrease in the amount of power shared by the prosumer. For example, the new rules to increase the incentives for the prosumers (external factor) adds positive weight to the total amount of energy shared and an increase in high wattage power loads (internal factor) can increase the amount of power consumed, thus adds negative weight to the energy shared with the grid.

\section{PROSUMER COMMUNITY FORMATION}

In this section, we present a prosumer-community formation model using multi-agent system. The multi agent system includes intelligent agents that autonomously interact with each other using decentralized data objects and asynchronous computation [30]. As illustrated in Fig. 3, we propose our system with two modules namely prosumer module and community operation centre module, which contains five agents and seven objects.

\section{A. Prosumer Home Module}

The prosumer home module contains power generation and consumption object and the prosumer agent. The power generation and consumption object maintains three tables namely prosumer identification data, prosumer current energy profile and prosumer historic energy profile (based on the internal factors discussed in section III). The prosumers can input their preferences and identification data to this object and also the energy data is being read from smart meters (supervised by energy retailers). The prosumer agent collects the data from above two sources and transmits to the community operation center through community coordination agent for processing.

\section{B. Community Operation Module}

The community operation centre module operates with four agents and six objects, which is made up of a community object and 5 external objects namely power market object, technical infrastructure object, weather forecast object, government jurisdiction object and general public object (object bases contain the external factors discussed in section III). The community object comprises prosumer-community prequalification criteria that highlight the community goals.

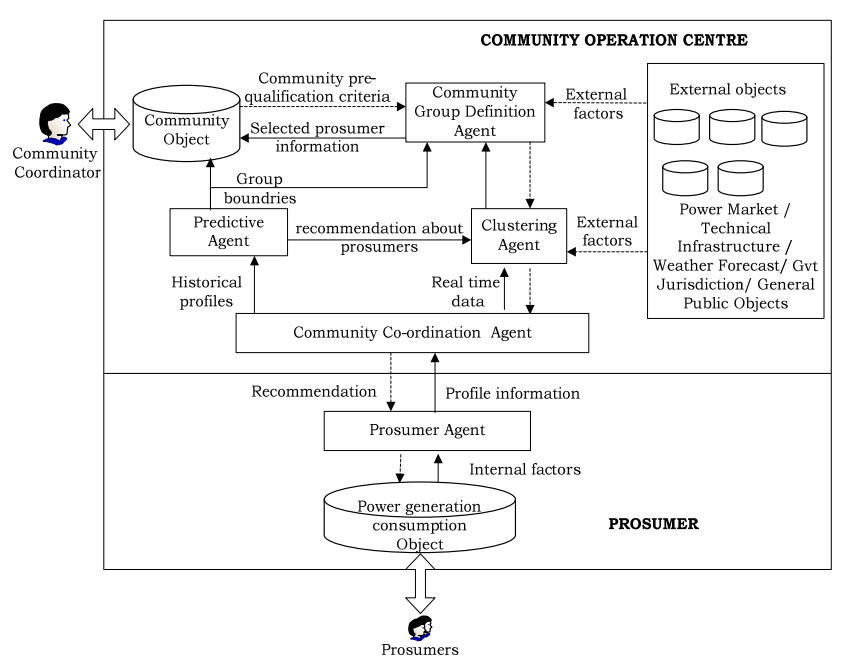

Fig.3. Multi Agent Architecture

Some of the community goals would be the maximum and minimum overall energy efficiencies to increase the community's overall profits, additional energy requirements to meet the energy variations caused by external factors, incentive levels assigned for prosumers, etc. Here we further discuss about the four intelligent agents.

1) Community coordinator agent: Community coordinator agent facilitates the communication between the two modules. It obtains the information from the prosumer agent and transfers to appropriate server or agent in community operation centre for further processing.

2) Predictive agent: Predictive agent analyses historic energy data of prosumers obtained from energy retailers and forecast the prosumers' future energy behaviours. The contribution of the predictive agent is two fold. First it offers recommendations on prosumers about their long-term capability of energy sharing. Since the prosumer energy data are stochastic and dynamic in nature, optimal sequential estimation techniques are required for prediction algorithm. Second contribution is that, it thoroughly analyses the prior data of prosumers and identifies the different minimum and maximum enrgy thresholds associated with prosumercommunity group definitions.

3) Community definition agent: Community definition agent defines the community-groups with suitable energy thresholds and also enforeces the rules to filter the suitable prosumers based on the community prequalification criteria and community goals located in community object. One of the most obvious rule would be the removal of the prosumers who are unable to provide at least the minimum expected energy level defined by the community -coordinator. These rules are defined at the community object, considering the suggestions obtained from external databases and other agents. 
4) Clustering agent: Based on both real-time energy profiles and recommendations obtained from predictive agent, the clustering agent filters the prosumers having similar energy behaviours, based on the community prequalification criteria in community object. There are a large set of both the categorical data like the geographical location of prosumers and continuous data like energy consumption and energy generation data. Therefore using hierarchical clustering that requires matrix of distances between all pairs of cases, and simple k-means algorithms that require shuffling cases in and out of clusters and predefining number of clusters in advance, are not very feasible with this. This highlights the necessity of having an optimal clustering technique, which needs preclustering to reduce the size of the distance matrix and then form the clusters, facilitating either to predefine the required number of clusters and or go for the optimal number of clusters selected by the algorithm.

\section{IMPLEMENTATION DETAILS}

If we consider the implementation details of the framework we will have a community smart gateway that will act as a point of contact for all the prosumers to the community coordinator and other external parties such as utility company [31-33]. The community smart gateway will have a unique IP address. Each internal smart gateway of a unit communicates with the smart gateway of the community which transmits the information to the community-coordinator. Based on the external and internal databases and community goals, the techniques for community formation and evolution are decided at the community smart gateway level which is them transmitted back to the smart gateway of each prosumer. The intelligent gateways will be realised through a multi-agent structures, which we discussed in the last section. The key protocol that coordinates all community gateways are the syndication protocol. We will employ the JXTA (Juxtapose) Peer-to-Peer framework to facilitate the federated cooperative service (i.e. utility information) discovery amongst these distributed community gateways. Further implementation details are out of the scope of this paper.

\section{FUTURE WORK}

Currently, this research project is in its initial stage. In future, we will develop relevant algorithms in order to build an intelligent framework for prosumer-community formation, and management. These algorithms would be; prosumer clustering algorithm to segment prosumers having similar energy behaviours, prosumer-community goal definition algorithm, prosumer-community formation algorithm, and prosumers' energy prediction algorithm followed by prosumer ranking algorithm in order to find out more dynamic prosumers within the entire prosumer base. Finally we will implement this entire system on top of web of things architecture [34].

\section{CONCLUSIONS}

In this article, we first suggest a novel concept of goaloriented virtual prosumer-communities in order to achieve more sustainable prosumer participation and management scheme. Then we analyse different parameters that directly and indirectly influence the energy sharing behaviours of prosumers, which is the initial step of achieving an effective prosumer-communities. Finally we present a multi-agent based model to form the optimal prosumer communities.

\section{REFERENCES}

[1] Energy outlook." Available: .http://www.eia.doe.gov/oiaf/ieo/world.html, accessed 05/04/2011

[2] "Smart Grid." Available: http://www.smartgrids.eu/?q=node/163, accessed 02/04/2011

[3] T. S. Dillon, A. Talevski, V. Potdar and E.Chang, "Web of things as a framework for ubiquitous intelligence and computing," in Proc. 2009 6th International Conference on Ubiquitous Intelligence and Computing, Brisbane, Australia, 2009, pp. 2-13.

[4] J. A. Momoh, "Smart grid design for efficient and flexible power networks operation and control," in Proc. 2009 Power Systems Conference and Exposition, pp. 1-8.

[5] A.J. D. Rathnayaka, V. M. Potdar, and S.Kuruppu, "Evaluation of wireless home automation technologies," presented at 5th IEEE International Conference on Digital Ecosystems and Technologies, Korea, 2011.

[6] C. Cecati, G. Mokryani, A. Piccolo and P. Siano, "An overview on the smart grid concept", in Proc. 2010 36th Annual Conference on IEEE Industrial Electronics Society(IECON 2010), pp. 3322-3327

[7] V. C. Gungor, L. Bin, and G. P. Hancke, "Opportunities and challenges of wireless sensor networks in smart grid," in Proc. IEEE Transactions on Industrial Electronics, vol. 57, no. 10, pp. 3557-3564, 2010.

[8] V. K. Sood, D. Fischer, J. M. Eklund and T. Brown, "Developing a communication infrastructure for the smart grid," in Proc. 2009 Electrical Power \& Energy Conference (EPEC), pp. 1-7.

[9] R. Belhomme, R. C. R. D. Asua, G. Valtorta, A. Paice, F. Bouffard, R. Rooth, and A. Losi, "ADDRESS - active demand for the smart grids of the future," IET Seminar Digests, vol. 2008, no. 12380, pp. 33, 2008.

[10] "Demand Response Project," Available: http://www.ieso.ca/imoweb/consult/demandresponse.asp.

[11] "iDEaS: Intelligent Decentralised Energy-Aware Systems," Available: http://www.ideasproject.info/index.php.

[12] P. Vytelingum, T.D.Voice, S.D.Ramchurn, A.Rogers and N.R.Jennings, "Agent-based micro-storage management for the smart grid," in Proc 2010 9th International Conference on Autonomous Agents and Multiagent Systems (AAMAS 2010), Toronto, Canada, pp. 39-46.

[13] Mihai, "Virtual power plants: A solution for diminishing the barriers for the penetration of uncontrollable resources", Energy Infrastructure Scenarios for the South-Eastern Europe, pp. 1-13, SUSPLAN-Regional Kick-Off Workshop, 2009.

[14] K. Dielmann, and A. van der Velden, "Virtual power plants (VPP) - a new perspective for energy generation?," in Proc.2003 9th International Scientific and Practical Conference of Students, Post-graduates and Young Scientists, pp. 18-20.

[15] D. Pudjianto, C. Ramsay, and G. Strbac, "Virtual power plant and system integration of distributed energy resources," IET Renewable Power Generation, vol. 1, no. 1, pp. 10-16, 2007.

[16] G. Chalkiadakis, V. Robu, R. Kota, A. Rogers and N. Jennings, "Cooperatives of distributed energy resources for efficient virtual $p$ plants," in Proc. $201110^{\text {th }}$ International Conference on Autonomous Agents and Multiagent Systems (AAMAS-2011), Taipei, Taiwan.

[17] A. L. Dimeas, and N. D. Hatziargyriou, "Agent based control of virtual power plants," in Proc. 2007 International Conference on Intelligent Systems Applications to Power Systems, ISAP 2007, 2007, pp. 1-6.

[18] P. C. Stern, "Information, incentives, and proenvironmental consumer behavior", Journal of Consumer Policy, vol. 22, no. 4, pp. 461-478, 1999.

[19] I. Lampropoulos, G. M. A. Vanalme, and W. L. Kling, "A methodology for modeling the behavior of electricity prosumers within the smart grid," in Proc. 2010 Innovative Smart Grid Technologies Conference Europe (ISGT Europe), pp. 1-8. 
[20] M. Miller, T. Batterberry, K. Jaskolka, and R. Toll, "Smart grid price response service for dynamically balancing energy supply and demand, ". US patent 0138363, USA, June, 1999.

[21] K. Kok, S. Karnouskos, D. Nestle, A. Dimeas, A. Weidlich, C. Warmer, P. Strauss, B. Buchholz, S. Drenkard, N. Hatziargyriou, and V. Lioliou, "Smart houses for a smart grid. Electricity Distribution - Part 1," in Proc. 2009 20th International Conference and Exhibition, pp. 1-4.

[22] C. W. Yeh, Wang, and C. Tseng. "iPower: an energy conservation system for intelligent buildings by wireless sensor networks," Int. J. Sen. Netw, vol. 5, no. 1, pp. 1-10, 2009.

[23] S. Tompros, N. Mouratidis, M. Caragiozidis, H. Hrasnica, and A. Gavras, "A pervasive network architecture featuring intelligent energy management of households," In Proc. 2008 1st international conference on PErvasive Technologies Related to Assistive Environments (PETRA '08), pp. 1-6.

[24] "Home energy consumption versus solar PV generation." Available: http://www.solarchoice.net.au/blog/home-energy-consumption-versussolar-pv-generation/, accessed 30/03/2011.

[25] "Vanadium Redox Batteries." Available: http://www.americanvanadium.com/vanadium-redox-batteries.php, accessed 15/05/2011.

[26] "Market Structure."Available: http://www.imowa.com.au/marketstructure, accessed 1/05/2011.

[27] "Electricity Auctions." http://energyauctions.com.au/?pageid=4, accessed 1/05/2011.
[28] "Office of Energy." Available http://www.energy.wa.gov.au/3/3621/64/system_owners.pm, accessed $1 / 06 / 2011$.

[29] "Metering." Available: http://www.westernpower.com.au/retailersgenerators/Metering.html, accessed 15/06/2011

[30] M. J. Wooldridge, An introduction to multiagent systems, 1st ed., 2009.

[31] A.M.A.H. Al-Kuwari, C. Ortega-Sanchez, C, A. Sharif, and V. Potdar, "User friendly smart home infrastructure: BeeHouse", in Proc. $20115^{\text {th }}$ IEEE International Conference on Digital Ecosystems and Technologies Conference (DEST), pp. 257 - 262.

[32] A.J.D. Rathnayaka, and V. Potdar, "Energy Resource Management in Smart Home: State of the Art and Challenges Ahead," in Proc. 2011 International Conference on Sustainability in Energy and Buildings (SEB 2011), Marseilles, France.

[33] K.T. Tejani, Al-Kuwari, and V. Potdar, "Energy conservation in a smart home," in Proc. 2011 IEEE International Conference on Digital Ecosystems and Technologies, Daejeon, Korea.

[34] T. Dillon, V. Potdar, J. Singh, and A. Talevski, "Cyber Physical Systems: Challenges in Sensor Actuator Networks," in Proc 2011 5th International Conference on Digital Ecosystems \& Technologies (DEST2011). 